POS PROCEEDINGS

\title{
Soft spectator scattering in the nucleon form factors at large $Q$ within the soft collinear effective theory approach
}

\author{
Nikolay Kivel \\ Helmholtz Institute, Mainz \\ E-mail: kivel@kph.uni-mainz.de
}

\begin{abstract}
The proton form factors at large momentum transfer are dominated by two contributions which are associated with the hard and soft rescattering respectively. Motivated by a very active experimental form factor program at intermediate values of momentum transfers, $Q^{2} \sim 5-15 \mathrm{GeV}^{2}$, where an understanding in terms of only a hard scattering mechanism cannot yet be expected, we investigate in this work the soft spectator scattering contribution using soft collinear effective theory (SCET). Within such description, the form factor is characterized, besides the hard scale $Q^{2}$, by a hard-collinear scale $Q \Lambda$, which arises due to presence of soft spectators, with virtuality $\Lambda^{2}(\Lambda \sim 0.3-0.5 \mathrm{GeV})$, such that $Q^{2} \gg Q \Lambda \gg \Lambda^{2}$. In case of nucleon FFs the soft spectator scattering mechanism contributes at leading power accuracy and therefore it must be considered in the systematic QCD factorization approach. We discuss how to generalize the well known Brodsky-Lepage collinear factorization in order to include the soft spectator contribution using the SCET technique. We carefully investigated the factorization of the soft and collinear modes in this case and demonstrate that even for the FF $F_{1}$ the pure collinear factorization could not be valid due to the mixing of the soft and collinear contributions. As a result this allows one to put specific constrains on the end-point behavior of the nucleon distribution amplitude.
\end{abstract}

Sixth International Conference on Quarks and Nuclear Physics

April 16-20, 2012

Ecole Polytechnique, Palaiseau, Paris 


\section{Introduction}

The leading power behavior of the FFs was studied a long time ago using the QCD factorization approach, see e.g. [1] and references therein. It was established that the dominant contribution can be represented by a reduced diagram as shown in Fig.1 (left) In this figure, the hard blob de-
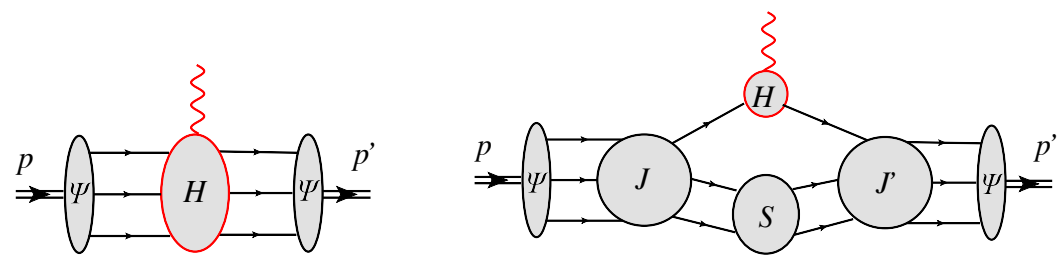

Figure 1: Reduced diagrams describing the hard and soft spectator scattering (left and right, respectively)

scribes the hard scattering of quarks and gluons with virtualities of order $Q^{2}$. Such hard subprocess can be systematically computed in perturbative QCD (pQCD) order-by-order. The soft blobs, denoted by $\Psi$, describe the soft, non-perturbative subprocesses, and can be parametrized in terms of universal matrix elements known as distribution amplitudes (DAs). Such picture suggests the well known factorization formula for the Dirac FF

$$
F_{1}=\int D x_{i} \int D y_{i} \Psi\left(x_{i}\right) \mathbf{H}\left(x_{i}, y_{i} \mid Q\right) \Psi\left(y_{i}\right) \equiv \Psi * \mathbf{H} * \Psi,
$$

and predicts the scaling behavior

$$
F_{1} \sim \frac{\Lambda^{4}}{Q^{4}} \times[\ln Q / \Lambda]^{\gamma}
$$

where the logarithmical corrections can be systematically computed order-by-order.

Unfortunately, for the Pauli FF $F_{2}$ this approach can not provide such systematic picture and suggests only the power estimate

$$
F_{2} \sim \frac{\Lambda^{6}}{Q^{6}}
$$

However already long time ago it was suggested [2] that the so-called soft spectator scattering mechanism, associated with the scattering of the hard virtual photon off one active quark, may dominate the nucleon FFs at moderate values of $Q^{2}$. The other spectators remain soft and therefore very often such scattering is associated with the soft overlap of the nucleon wave functions. Later it was found that the picture described by the first diagram in Fig.1 is not complete. In Ref. [3] it was demonstrated that the exchange of soft quarks between initial and final states may also produce contribution of order $1 / Q^{4}$ accompanied by large logarithm. At the same time, many phenomenological studies support the conclusion that in the region of moderate $Q^{2} \simeq 5-10 \mathrm{GeV}^{2}$ it is difficult to describe the FF data using only the factorization approach expressed by Eq. (1.1). Recent measurements of the FF ratio $F_{2} / F_{1}$ up to $Q^{2}=8.5 \mathrm{GeV}^{2}$ [4] also do not support the expectation of Eq. (1.3) which assumes that in the asymptotic region $Q^{2} F_{2} / F_{1} \sim$ const up to logarithmic corrections. It allows to expect that contribution from the soft spectator scattering can be large. Such picture is supported by different phenomenological approaches, such as QCD-motivated models for the hadronic wave functions [5] and QCD sum rules [6]. 
In the first paper [7] we suggested to apply the effective theory approach, known as soft collinear effective theory (SCET), in order to describe consistently both contributions associated with the hard and soft spectator scattering within QCD factorization approach. In [8] we carried out more detailed investigation of the suggested factorization formula for the FF $F_{1}$. Below we shortly describe obtained results.

\section{Nucleon FFs within SCET factorization approach}

The specific feature of the soft spectator scattering is the presence of two subprocesses related to the two hard scales: a hard subprocess with typical scale of order $Q^{2}$ and a hard-collinear subprocess with typical scale of order $\Lambda Q$. A description of such processes could be carried out in two steps: first, one integrates over hard fluctuations so that the remaining degrees of freedom describe hard-collinear and soft processes. Therefore one needs the effective theory describing the dynamics of such system. Such effective theory, known as soft collinear effective theory (SCET), was built already for the description of heavy quark decays and some other hadronic reactions [9, 10].

If $Q$ is large enough, so that the hard collinear scale $\sim Q \Lambda$ can be considered as a good expansion parameter in $\mathrm{QCD}$, one can further use perturbation theory and factorize the hard-collinear fluctuations, leaving at the end only collinear and soft modes which describe soft QCD dynamics. Technically, such two step factorization is described as matching of full QCD onto the soft collinear effective theory at the scale $\mu=Q$ (SCET-I), that is equivalent to calculating the hard coefficient functions in front of an operator constructed from SCET-I fields described above. The second step is the matching of SCET-I at the scale $\mu=\sqrt{\Lambda Q}$ to SCET-II, that again corresponds to the pQCD calculation of hard-collinear coefficient functions (which are usually called jet functions) in front of operators constructed only from the collinear and soft fields.

Following this scheme we obtain that the full description of large- $Q$ behavior of the nucleon FF $F_{1}$ is given by the sum of two contributions associated with the soft and hard rescattering picture:

$$
F_{1} \simeq F_{1}^{(s)}+F_{1}^{(h)}
$$

The hard rescattering part $F_{1}^{(h)}$ is well known and described by (1.1). One can expect that the soft contribution can also be presented in a factorized form but with the more complicated structure reflecting the presence of different scales. Performing the leading logarithmic analysis of the leading power contribution $\left(\sim 1 / Q^{4}\right)$ we suggested the following tentative description of the soft spectator contribution [7]:

$$
F_{1}^{(s)} \simeq C_{h}(Q) \int_{0}^{\infty} d \omega_{1} d \omega_{2} \Psi\left(y_{i}\right) * \mathbf{J}^{\prime}\left(y_{i}, \omega_{i} Q\right) \int_{0}^{\infty} d v_{1} d v_{2} \Psi\left(x_{i}\right) * \mathbf{J}\left(x_{i}, v_{i} Q\right) \mathbf{S}\left(\omega_{i}, v_{i}\right),
$$

which can be interpreted in terms of reduced diagram as in Fig.1(right). This result involves a hard coefficient function $C_{h}$, and two hard-collinear jet functions $\mathbf{J}$ and $\mathbf{J}^{\prime}$ which can be computed in pQCD. They describe the subprocesses with hard momenta and hard collinear momenta respectively. The non-perturbative functions $\Psi$ and $S$ describe the scattering of collinear and soft modes. The convolution integrals in Eq. (2.2) are performed with respect to the collinear fractions $x_{i}$ and $y_{i}$, and with respect to the soft fractions $\omega_{i}, v_{i} \sim \Lambda$. Explicit definitions of the soft functions can be fond in $[7,8]$. 
We expect that the similar factorization scheme is valid also in the case of the Pauli FF $F_{2}$. The difference is that one needs to involve subleading SCET operators in order to describe properly the matching to SCET-I, see details in [7].

If the value of $Q$ is moderate, (for instance, the hard-collinear scale $Q \Lambda \sim m_{N} \sim 1 \mathrm{GeV}^{2}$ is not large in order to serve as expansion parameter) then one can not perform SCET-II factorization. Phenomenologically such situation is relevant for quite a large range of experimentally accessible values of momentum transfer. For instance, if $Q^{2}$ is in the range of $4-25 \mathrm{GeV}^{2}$ one obtains that $Q \Lambda$ varies between $0.8-2 \mathrm{GeV}^{2}$ assuming that $\Lambda \simeq 400 \mathrm{MeV}$. In this case the factorization can be performed only in terms of the SCET-I matrix elements:

$$
\begin{aligned}
& F_{1}^{(s)}\left(Q^{2}\right)=C_{A}(Q, \mu) \sum_{q} e_{q} f_{1}^{q}(Q, \mu), \\
& F_{2}^{(s)}\left(Q^{2}\right)=\frac{m_{N}^{2}}{Q^{2}} \int_{0}^{1} d \tau C_{B}(\tau, Q, \mu) \sum_{q} e_{q} f_{2}^{q}(\tau ; Q, \mu)+\frac{4 m_{N}^{2}}{Q^{2}} C_{A}(Q, \mu) \sum_{q} e_{q} f_{1}^{q}(Q, \mu) .
\end{aligned}
$$

where $C_{A, B}$ denotes the hard coefficient functions ${ }^{1}$. The SCET-I FFs $f_{1,2}$ are defined in SCET as a matrix elements of SCET operators. For instance,

$$
\left\langle p^{\prime}\left|\bar{\xi}_{q}^{\prime} W^{\prime} \gamma_{\perp}^{\mu} W^{\dagger} \xi_{q}\right| p\right\rangle_{\mathrm{SCET}}=\bar{N}\left(p^{\prime}\right) \frac{\bar{n} n}{4} \gamma_{\perp}^{\mu} \frac{\bar{n} n}{4} N(p) f_{1}^{q}(Q, \mu)
$$

where $\bar{\xi}_{q}^{\prime} W^{\prime} \gamma_{\perp}^{\mu} W^{\dagger} \xi_{q}$ represent the leading order SCET operator constructed from the hard-collinear fields, $n$ and $\bar{n}$ denote the convenient light-cone vectors associated with nucleon momenta $p$ and $p^{\prime}$, see details in [7]. FFs $f_{2}^{q}$ are defined by the subleading SCET operator and therefore they depend on the light-cone fraction $\tau$ describing how the longitudinal momentum is shared between the constituents inside the hard-collinear jet. From the physical point of view the SCET FFs describe the interaction of the quark-gluon hard-collinear jets with the soft background. Therefore SCET FFs depend only on the hard-collinear virtualities $\sim Q \Lambda$ (which we indicate for simplicity as Q) and factorization scale $\mu$. Dependence on this scale can be computed from the renormalization of the SCET-I operators. Perturbative resummations of large logarithms in the coefficient functions can be done using RG equations and include also the well-known Sudakov logarithms.

In [8] more detailed analysis of suggested factorization has been carried out. Using computed leading order jet functions $J$ appearing in the matching from SCET-I to SCET-II we obtained that the corresponding soft convolution integrals in definition $F_{1}^{(s)}(2.2)$ have logarithmic divergency at the end-point region.

We suggested that the appearance of end-point singularity can be explained by overlap of collinear and soft regions. In order to verify this supposition we carried out analysis of two-loop QCD diagrams with massive quarks and investigated the dominant contributions associated with the different regions. We found that the overlap of the soft and collinear sectors provides the large rapidity logarithm $\sim \ln Q / m$ where $m$ is the quark mass which plays the role of the soft scale. Thus the logarithmic divergence of the soft convolution integrals can be naturally explained by the overlap with the collinear region. In such case the collinear convolution integrals describing the hard spectator scattering has also end-point singularity and can not be well defined without special

\footnotetext{
${ }^{1}$ The second term with $f_{1}$ in Eq.(2.4) for $F_{2}^{(s)}$ is the kinematical correction. This term has been overlooked in [7].
} 
regularization. The latter allows us to conclude that the separation of the hard and soft spectator contributions for the FF $F_{1}$ is closely related to the problem of separation of soft and collinear sectors in SCET.

At first glance such situation looks controversial for the FF $F_{1}$ taking into account the endpoint behavior of the nucleon DA which is usually estimated from the QCD evolution. The eigenfunctions of the the leading order evolution kernel vanish quite rapidly at the end-point region that ensures a good convergence of the collinear integrals defining the hard spectator contribution. However the soft spectator scattering and explicit chiral symmetry breaking (mass of the soft quarks) in perturbative calculations provides the mechanism which makes possible the soft collinear overlap and violation of the collinear factorization without any conflict with these arguments. QCD evolution is not sensitive to the effects related to the mass of quarks and therefore in this case it can not provide a signal about the problem with the end-point behavior of the collinear convolution integrals. Formally, in the perturbation theory this scenario is realized as following. The end-point behavior of the DA at low normalization $\left(\mu_{F}=m\right)$ is different from the one which we expect from its evolution: it vanishes more slowly. Then the convolution integral with such DA has logarithmic end-point divergency.

Extrapolating these arguments beyond the perturbation theory we can not use the mass of the soft quarks as realistic argument anymore. But on the other hand we can expect that the nontrivial contribution to the soft correlation function $\mathbf{S}$ in Eq.(2.2) can be obtained due to the dynamical chiral symmetry breaking in non-perturbative QCD. In that case one can have the nontrivial soft spectator scattering contribution which has the end-point singularities. Basing on this observation we can formulate the following conjecture. The soft spectator scattering and chiral symmetry breaking in non-perturbative QCD make possible the soft collinear overlap and as a result violation of the collinear factorization for FF $F_{1}$. This leads to the appearing of the end-point singularities in the soft and hard spectator scattering contributions. In this case the nucleon DA $\varphi_{N}\left(x_{i}, \mu_{0}\right)$ at a low normalization point $\mu_{0} \sim \Lambda$ vanishes more slowly then the asymptotic shape and such behavior leads to the end-point singularities in the collinear convolution integrals. The end-point singularity arising in the soft spectator contribution can be considered as strong argument in support of such scenario.

\section{References}

[1] G. P. Lepage and S. J. Brodsky, Phys. Rev. D 22 (1980) 2157, V. L. Chernyak and A. R. Zhitnitsky, Phys. Rept. 112, 173 (1984).

[2] R. P. Feynman, "Photon-Hadron Interactions," Reading, 1972, 282p.

[3] A. Duncan and A. H. Mueller, Phys. Rev. D 21, 1636 (1980).

[4] A. J. R. Puckett et al., Phys. Rev. Lett. 104, 242301 (2010).

[5] N. Isgur and C. H. Llewellyn Smith, Phys. Rev. Lett. 52, 1080 (1984).

[6] B. L. Ioffe and A. V. Smilga, Nucl. Phys. B 216, 373 (1983), V. A. Nesterenko and A. V. Radyushkin, Phys. Lett. B 115, 410 (1982), V. M. Braun, A. Lenz and M. Wittmann, Phys. Rev. D 73, 094019 (2006).

[7] N. Kivel and M. Vanderhaeghen, Phys. Rev. D 83 (2011) 093005 [arXiv:1010.5314]. 
[8] N. Kivel, arXiv:1202.4944 [hep-ph].

[9] C. W. Bauer, S. Fleming and M. E. Luke, Phys. Rev. D 63, 014006 (2000), C. W. Bauer, S. Fleming, D. Pirjol and I. W. Stewart, Phys. Rev. D 63, 114020 (2001), C. W. Bauer and I. W. Stewart, Phys. Lett. B 516, 134 (2001), C. W. Bauer, D. Pirjol and I. W. Stewart, Phys. Rev. D 65, 054022 (2002).

[10] M. Beneke, A. P. Chapovsky, M. Diehl and T. Feldmann, Nucl. Phys. B 643, 431 (2002), M. Beneke and T. Feldmann, Phys. Lett. B 553, 267 (2003). 